\title{
Proposal to apply a code of good teaching practice in engineering
}

\author{
Esther Sainz-Martín, Raúl Igual-Catalán, Inmaculada Plaza-García Senior Member IEEE, Iván García- \\ Magariño, Francisco Ibañez-Álvarez, Francisco Arcega-Solsona Senior Member IEEE.
}

\begin{abstract}
Excellence is one of the goals of Higher Education. One possible way to achieve this objective is by applying a culture based on the concepts of quality and innovation. However, the real-world application of these concepts in Higher Education teaching is a difficult task. In this paper, we present a Web tool to help teachers apply a code of good teaching practice based on the aforementioned concepts. This code has been used for several years in Electrical Engineering, Electronics and Computing courses, although it could be applied to any other area. To assess this code, objective and external metrics have been used. Results show that the code helps to improve students' satisfaction as well as their academic results. Teachers' views on the code are also moderately positive.
\end{abstract}

Index Terms - Real-world evaluation, good practice, teaching, quality, innovation, engineering.

\section{INTRODUCTION}

$\mathrm{T}$ he concept of excellence has acquired great relevance in the university environment. Excellence in this field can be analyzed from different perspectives [1]. Although they can be considered separately, all of them have important points of convergence. In this regard, we can distinguish among excellence in teaching, excellence in university management and excellence in research and knowledge transfer. Although university excellence includes all processes involved in the educational activity and covers all grades and levels, teaching excellence is more related to the teaching-learning process in the classrooms and laboratories (either virtual or physical).

The concept of excellence is closely related to the concepts of quality and innovation. Thus, excellence in the academic environment can be achieved through the adoption of a culture that focuses on them.

E.S., R.I., F.I. y F.A. belong to the I+D+I EduQTech Group (Education, Quality, Technology), Department of Electrical Engineering, EINA / EUPT, University of Zaragoza (e-mail: [esainz, rigual, fcoiba, arcegafj]@unizar.es).

I.P. belongs to the I+D+I EduQTech Group (Education, Quality, Technology), Department of Electronics Engineering and Communications, Polytechnic University School of Teruel (EUPT), University of Zaragoza, E44003, Teruel (e-mail: inmap@unizar.es).

I.G-M. belongs to the I+D+I EduQTech Group (Education, Quality, Technology), Department of Computer Science and Systems Engineering, Polytechnic University School of Teruel (EUPT), University of Zaragoza, E44003, Teruel (e-mail: ivangmg@unizar.es).
With respect to the quality concept, one of the most common definitions is provided by Ishikawa [2]. He defines it as "philosophy, culture, strategy or management style of a company, in which all people study, practice, participate and promote the quality continuous improvement". According to this definition, the application of quality concepts in the university environment does not only refer to students or the teaching-learning process but also involves teachers' selforganization, universities and faculties' structure, all types of human resources (students, teachers, administrative staff), as well as society in general. Thus, quality cannot be considered in a limited way. Instead, an integral perspective must be provided, following the guidelines of all-pervasive quality [2].

As regards the innovation concept, Anttila [3] relates it to new viable solutions for products, processes, business systems or technologies that can be adopted by companies, governments or society in general. In other words, innovation can be seen as improved quality.

Although the concept of innovation has been traditionally associated with the business environment, it can also be applied to other disciplines such as education. In this regard, one of the tools to improve education quality is the use of innovative activities.

However, teachers interested in this area do not usually know how to apply quality and innovation concepts in their daily activities. The answer to this question is not simple, but a first approximation is to use popular standards about organization management (SQuaRE, ISO / IEC 19796, UNEISO 10002: 2004, UNE 66167: 2005, UNE 66173 IN: 2003 UNE 66175: 2003 and UNE 66178: 2004) [4]. The main difficulty lies in the way to apply these standards to the education field. One possible way is through a code of good teaching practice based on quality and innovation concepts [5].

However, the practical application of this code can be a challenge for university teachers. Its use in a real-world environment presents difficulties, since the code consists of different categories and processes, document management tasks and data collection activities. Therefore, in order to facilitate the practical application of this code, it is necessary to have a support tool that helps teachers in their daily work. In this paper, we present a Web-based software tool that implements the code of good teaching practice based on quality and innovation concepts and facilitates its practical 
use.

The rest of the paper is organized as follows: a review of studies in this area is included in section 2; section 3 describes the code of good teaching practice based on quality and innovation concepts; section 4 presents the methodology followed to implement the software application to support the use of the code; section 5 presents the structure of the Web platform; the use of the tool is explained in section 6; section 7 presents a preliminary real-world evaluation of the code; and finally, section 8 draws several conclusions and outlines areas for future research.

\section{RELATED WORK}

The results of the literature search present two different trends in existing studies [5].

On the one hand, an important part of the literature focuses on the application of quality concepts in education. Thus, in Rexwinkel et al. [6], the factors that determine quality in university education are identified. Meanwhile, Biggs [7] proposes a new educational approach called "constructive alignment", in which learning is based on the accumulated personal experiences of students as a result of their actions. Teachers' work is to propose activities to achieve specific learning goals. All these ideas are related to a quality-based planning. There are also studies that put forward models to manage and evaluate the quality of university education, such as Chen et al [8], which uses the Plan-Do-Check-Act cycle. Another set of studies focuses on evaluating the complexity of applying quality systems in university environments. Remarkable works are those of Jethro et al. [9] and Pratasavitskaya et al. [10], which highlight the difficulties associated with the implementation of quality systems. They identify numerous existing factors and models with no connections among them. This hinders the management and the integration of quality systems.

On the other hand, there are studies centred on innovation activities in higher education. An example of this trend is reflected in the work of Laurillard [11], which describes innovative activities in the university environment based on the use and design of learning technologies. Meanwhile, other studies highlight both the importance of including education in innovation as a key element to enhance talent $[12,13]$, and the importance of human capital for innovative environments [14]. There are also other studies that expand the perspective, and bring innovation to all aspects of higher education. They also provide some guidelines for promoting innovation in education. What all these studies have in common is that they consider the complexity associated with the implementation of innovative processes in education.

Despite the close relation between the concepts of quality and innovation in education, there are very few studies which treat them jointly [15]. In this regard, the work of Militaru et al. [16] analyses the quality and innovation factors that play an important role in the sustainability of the education system. These factors are related to creativity and generation of new ideas. Thus a conceptual model for sustainable higher education based on the union of ethics, quality, creativity and innovation is proposed. Furthermore, Van Kemenade et al.
[17] argue that universities should be less dependent on governments and should have a greater presence of quality products and services through the application of new organizational forms and the promotion of the entrepreneurial spirit.

All these works focus on applying simultaneously the concepts of quality and innovation in university education. However, none of them proposes the use of digital tools to manage the application of these concepts since they target theoretical formulation of models rather than real-world implementation. For this reason and considering that the application of these concepts is a complex process, there is a need for a software tool that facilitates systematization and real-world use.

\section{Code of Good TeAching Practice}

The code of good practice developed states the points and methods to be considered in all areas of teaching: planning, daily teaching, evaluation, teaching continues improvement, etc. It also incorporates the concepts of quality and innovation in all stages in order to conform to existing standards as much as possible.

As both quality and innovation have been regulated through standards, the UNE 66931 standard has been considered as the basis, adopting a process-based approach. According to this standard, it is essential to identify the processes of the code and the relations among them. In this regard, the processes involved in this code are grouped into three categories, following the proposals of Hines and Taylor [18]. These categories are: operational processes, strategic processes and support processes.

For each one of these categories, several associated processes have been defined which cover the entire educational cycle. Thus, the processes associated with the category of operational processes are: specific planning, teaching, evaluation and closing of the school year. As for the strategic processes category, the following processes have been identified: management, advanced planning and continuous improvement. Finally, the support processes group includes the following: resource management, documentation management, problem solving and quality.

The processes and the relations among them are represented in a process map. In addition, each process is described in a document called process sheet, which includes the following points: person in charge of the process, activities, records, resources, relations with other processes, etc. A graphical summary of the process sheets is contained in the flow diagrams, which include the most relevant information schematically.

All these elements represent and describe the code. However, the practical application of the code in daily teaching is done by filling in a set of records. Each process has several associated records. A record is a document which contains several fields to confirm that the code of good teaching practice is being applied and to ensure that it is correct. Therefore, the process map, flow diagrams, process 
sheets and records make up the code of good teaching practice based on quality and innovation concepts (Fig. 1). This code has already been applied for several years in courses of Electrical and Electronics Engineering. As a result of this experience, it was found that the practical application of the code can be hard and time-consuming. Thus, a computer tool is required that helps in the daily use of the code in university environments.

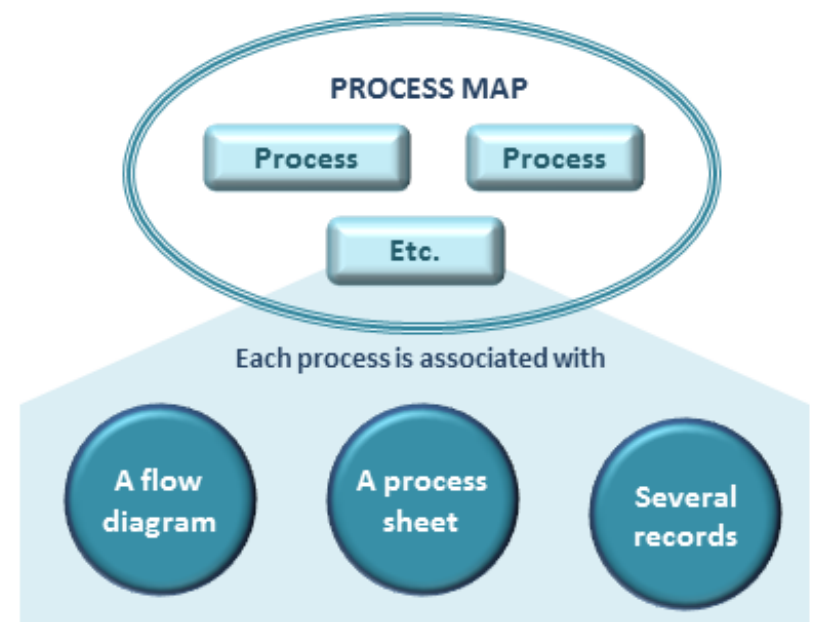

Fig. 1. Structure of the code of good teaching practice.

\section{Methodology}

In order to facilitate the daily use of the code, a software application has been developed. The application includes all levels of the code: from the process map to the records management. This application aims to: (i) facilitate that teachers of any institution have access to the code, (ii) improve code intelligibility and (iii) provide a support tool for the daily application of the code [5]. For the design of the tool, several principles regarding both the architecture of the application and its real-world use have been considered. These principles are:

1) Universality: The tool is intended for teachers of any area.

2) Ease of use: As this tool can be applied in the teaching of any discipline, usability recommendations have been followed for its design [5]. For example, all pages follow the same pattern, each process includes a graphic and text description, the colours of the application are intended to ease the use, etc.

3) Standardization: The application has been designed according to quality and innovation standards [5].

4) Accessibility: A Web application has been designed in order to be accessed from any place with Internet connection.

5) Privacy: The Web application includes a public area with the code information and a private area in which teachers can upload and manage their custom records.

6) Customization: The application supports different file formats. It also provides several record templates. Teachers may choose to use them or include their own personal formats. Thus, the application can adapt to each particular case.

\section{ApplicAtion ARCHitecture}

The application to manage the code of good practice has been developed following the Model-View-Controller (MVC) design pattern [19]. In this way, a dissociation of the layers forming the platform is achieved. The function of each layer is the following [5]:

- Model: The model is responsible for managing the storage of data (records in this case).

- View: The view is the application interface with the user.

- Controller: The controller is the central layer of the system. It receives the requests from users, asks the model for the requested information and shows it back to the user.

The combination of these three layers results in the final Web application: the Web-based code of good practice based on quality and innovation concepts.

\section{Use of the Code of Good Teaching Practice}

This section describes the Web tool to support the application of the code of good practice [5]. Fig. 2 represents a general diagram of the Web application. The central part of the scheme includes the home page. From this page, different sub-pages related to each one of the processes are derived. On a third level, associated with each page of the processes, there is a new set of pages in which teachers can store the records related to the processes.

Firstly, when a user accesses the application, he/she can see the process map, which represents all code processes and their relations (Fig. 3).

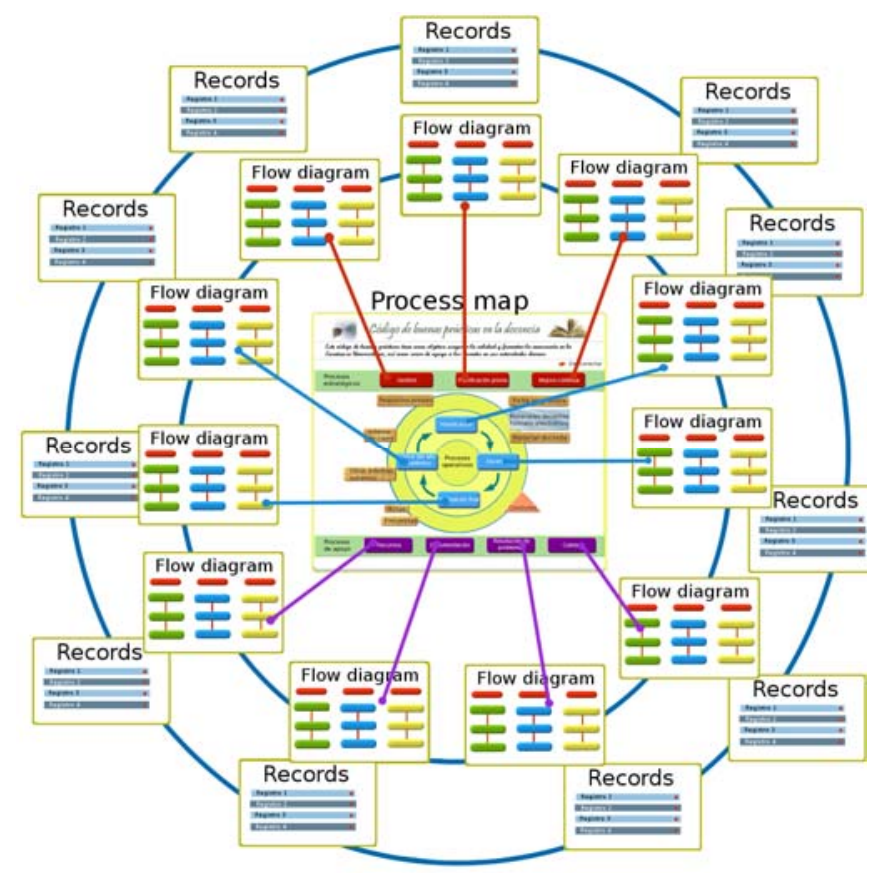

Fig. 2. General structure of the support Web application. 


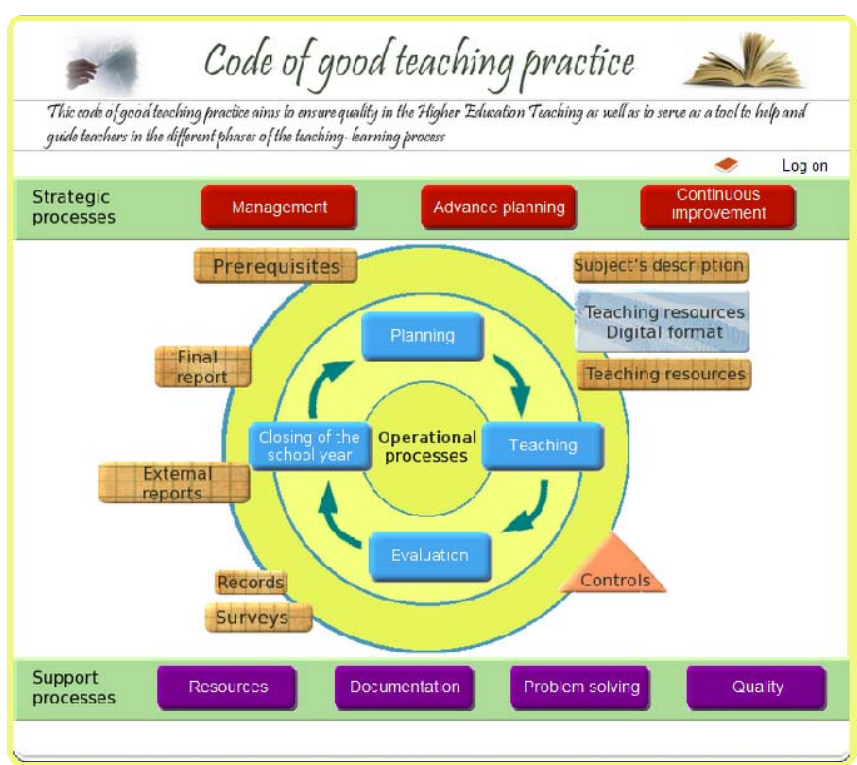

Fig. 3. Homepage of the application with the process map.

The processes are grouped into the three categories previously described. Teachers can click on each of them, and the application displays the page associated with the process (Fig. 4). Each page has a header with a title, a brief description, a link to download the process sheet with the detailed description, a flowchart and a set of records to be filled in by teachers.

By filling in those records, teachers can systematize and document the application of the code. The documentation management is done in the private part of the application, which requires previous authentication (Figure 5). In this area, teachers can download the templates associated with each record, fill them in and save them in the application. They can also list, download, edit and delete the records stored. Thus, the application includes a documentation management system associated with the real-world use of the code. Consequently, teachers are provided with a support tool for an efficient application of the code in daily teaching, minimizing the efforts required.

It is also worth mentioning that this tool provides high levels of flexibility in order to adapt to the teachers' needs. Thus, if a particular teacher considers that a process is not applicable to a specific context, he/she can avoid its use by not filling in the associated records. Similarly, if a particular teacher considers that the proposed records do not match his/her needs, he/she can replace them with personalized ones. These new records can be included in the personal private part of the tool. Additionally, the records uploaded can be modified at any time.

In order to use the support Web application, teachers should plan their course strategy. To this end, they should consider the processes that have been defined as strategic: management, advanced planning and continuous improvement. Then, a common case for a school year would be determined by the operational processes: specific planning, teaching, evaluation and closing of the school year. These processes have to be accompanied by the support processes: resource management, documentation management, problem solving and quality.

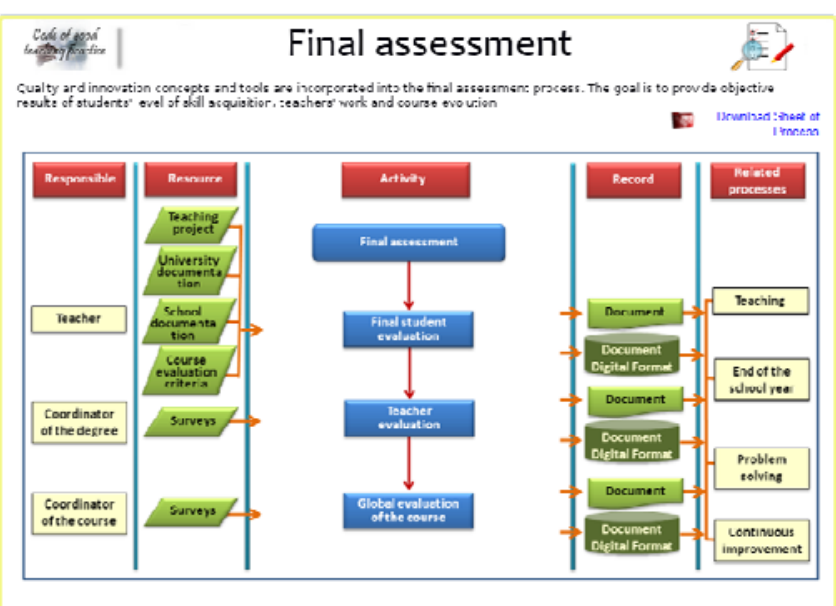

Fig. 4. Screenshot of the application flow chart associated with the "Final Evaluation" process. From this screen, teachers can download the process sheet (top right link) and the corresponding records.

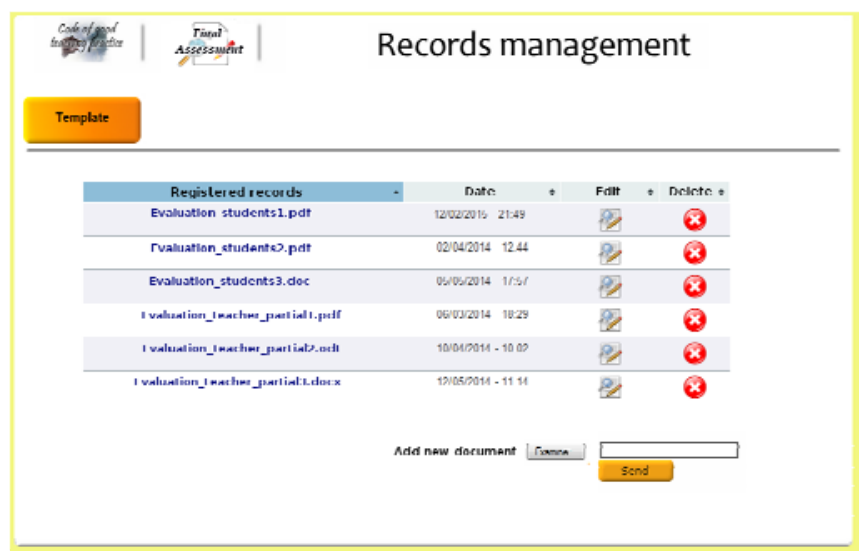

Fig. 5. Private part of the application with different records (shown as an example) associated with a particular teacher. Records are related to the "Final Evaluation" process.

\section{Evaluation of the Code OF GoOd Teaching PRACTICE}

As a real case study, this code of good practice has been applied to the "Fundamentals of Digital Systems" (FSD) course of the Degree in Computer Engineering at the University of Zaragoza (Teruel, Spain) [20]. FSD is a 60-hour course, 40 hours of classroom sessions and 20 hours of practical problem solving. The main objective of the course is to "provide the knowledge and skills needed to understand and use modern digital electronics, which is the basis of computer technology" [15].

In order to evaluate the application of this code in FSD, quality criteria have been used. In this regard, external and objective data obtained from different assessment tools have been considered. Specifically, these sources are [15]:

- The institutional external evaluation conducted by the "Commission of Teaching for the Evaluation of Educational Activities" of the University of Zaragoza. At 
the end of each semester, a member of the School board distributes a set of questionnaires to students in order to assess different teaching aspects. Each item is evaluated with a score from 1 (very poor) to 5 (outstanding). The University Teaching Commission processes the results and sends them to teachers.

- Students’ academic performance.

The code of good practice has been evaluated using these sources. As the final goal is to improve the quality of teaching, which is related to the students' perceptions of the course, several aspects associated with students' satisfaction and academic performance have been considered. This evaluation was performed in two different periods of the school year:

- Before applying the code of good practice in daily teaching.

- After applying the code of good practice.

Specifically, the aspects that have been evaluated are:

A) Students'satisfaction: Students' perceptions of teachers' knowledge of the course and students' perceptions of the teaching activities.

C) Lesson preparation.

D) Academic performance: Performance rate (percentage of enrolled students who passed the course) and Success rate (percentage of enrolled students attending the classes who passed the course).

Additionally, as both the implementation of the code and the use of the support Web application involve teachers, a preliminary assessment has also been conducted to determine whether or not the Web application represents an improvement in the practical use of the code. Six teachers were involved in the evaluation. They belonged to the Departments of Electrical Engineering, Electronics and Communications and Computer Science. All teachers had prior experience in the application of the code without web support. In order to carry out the assessment, the System Usability Scale (SUS) has been used [21]. This is an internationally accepted model to assess the degree of usability of a tool taking into account users' feedback. Specifically, the SUS questionnaire included ten questions evaluating five items. It has two questions related to each item, so that each question addresses the same idea in an antagonistic way:

- Satisfaction: A) I think I would use this application frequently / B) I found the application unnecessarily complex.

- Ease of use: A) I thought that the application is easy to use / B) I thought that the application was difficult to use.

- Integration: A) I found the different functions of this application well-integrated / B) I thought that there was too much inconsistency in this application.

- Learning: A) I think that most people learn how to use this application very fast / B) I think that I need support from technicians to use this application.
- Security: A) I feel very safe with the application / B) I have to learn many things before I can handle this application.

Each question is rated on a 1-5 scale where 1 means "Strongly Disagree" and 5 means "Strongly agree" in questions A of each item, and just the opposite in questions B. Once teachers filled in the questionnaires, the average rating for each item was calculated, and the equivalent value on the 0-100 scale was obtained. This value has to be interpreted according to Fig. 6.

Next subsections analyze the evaluation results.

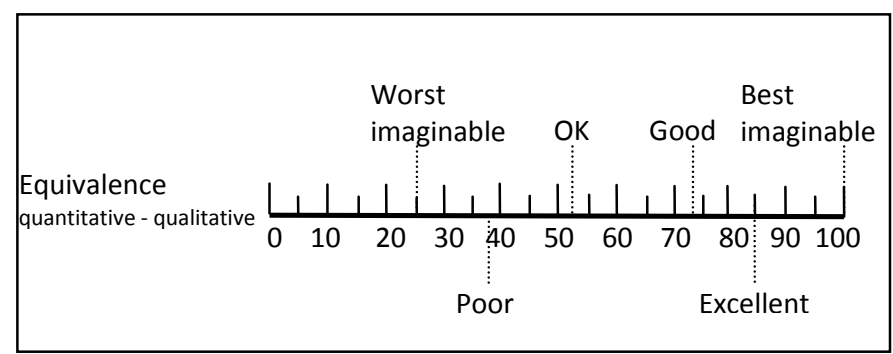

Fig. 6. Qualitative equivalency of SUS quantitative scale.

\section{A. Student satisfaction}

As a result of applying the code of good practice based on quality and innovation concepts, the overall level of students' satisfaction increased. Figure 7 shows the students' assessment of the item "Perception of the teacher's knowledge about the course" before and after applying the code of good practice. The results show that the application of the code had a direct effect on the students' perceptions of teachers' level of mastery about the course. In fact, students gave top marks to this item.

As a complement to this analysis, Fig. 8 presents students' overall level of satisfaction. The results clearly show that the level of satisfaction increased. Students' scores are between 4 and 5 after introducing the code.

\section{B. Lesson preparation}

Fig. 9 shows the results of evaluating the item "Lesson preparation" As in the previous cases, a clear improvement can be observed. In this regard, it is possible to conclude that, as a result of systematizing the teaching activities, the planning of the course improved, which was clearly perceived by students.

\section{Academic results}

Fig. 10 shows the evolution of two academic metrics (success rate and performance rate). In view of the results, it is possible to conclude that the two indicators improved after applying the code in daily practice. This increase was particularly significant in the success rate, reaching $15.3 \%$ of improvement.

\section{Preliminary assessment of the Web application}

Fig. 11 shows the results of the SUS questionnaires filled in by teachers to evaluate the support Web application. 
Questions related to the satisfaction with the use of the tool, the integration of its functions and the ease of the learning process achieved a rating in the range of "Good" according to the SUS scale. The most highly valued feature was the ease of use, in the range of "Excellent", while the perceived security in use was less highly valued, in the range of "Acceptable".

Additionally, teachers elaborated a list with the advantages of using the Web application to apply the code in daily teaching. Among other points, they highlighted the ease in organizing information, the time optimization, the possibility to access the code records from any place and the positive contribution to the easy elaboration of the reports to renew the official accreditations of the Degree and Master studies.

They also made another list with the aspects to be improved. Most teachers stressed the need to include the option of sharing documents between several teachers as well as between various processes.

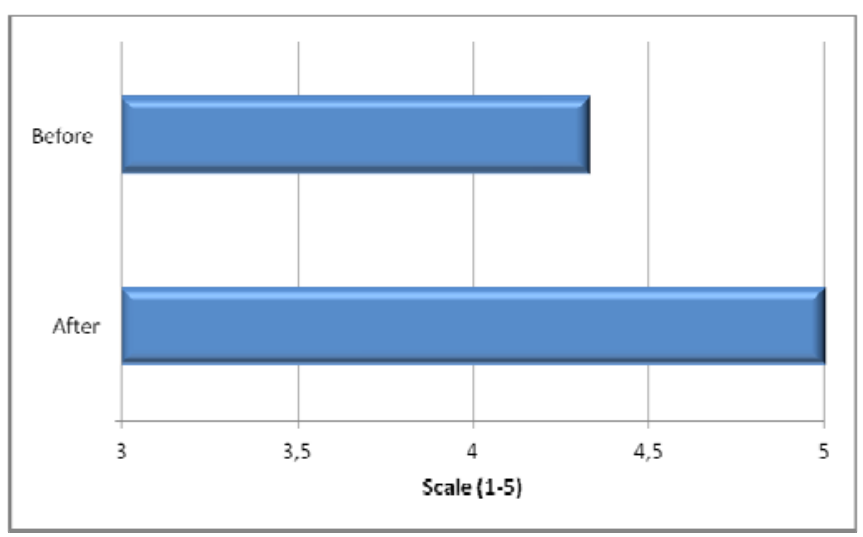

Fig. 7. Teachers' level of knowledge of the course on a 1-5 scale.

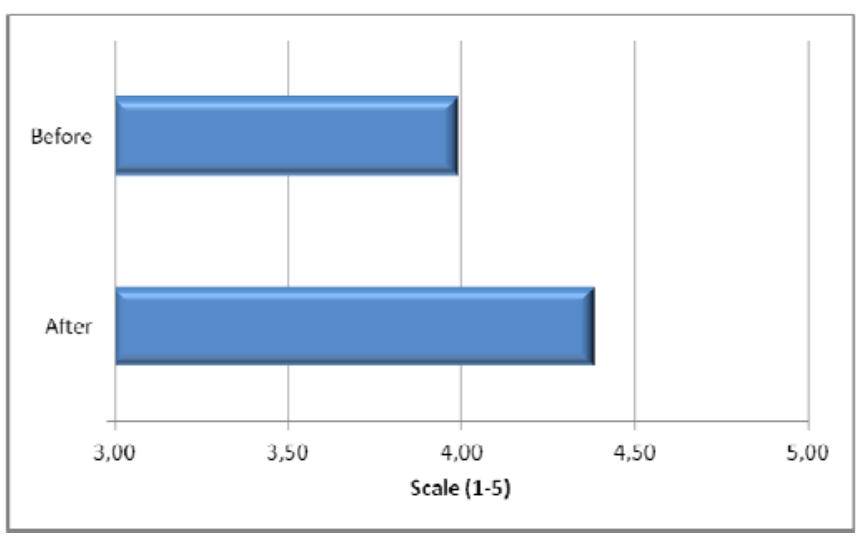

Fig. 8. Overall level of students' satisfaction on a 1-5 scale.

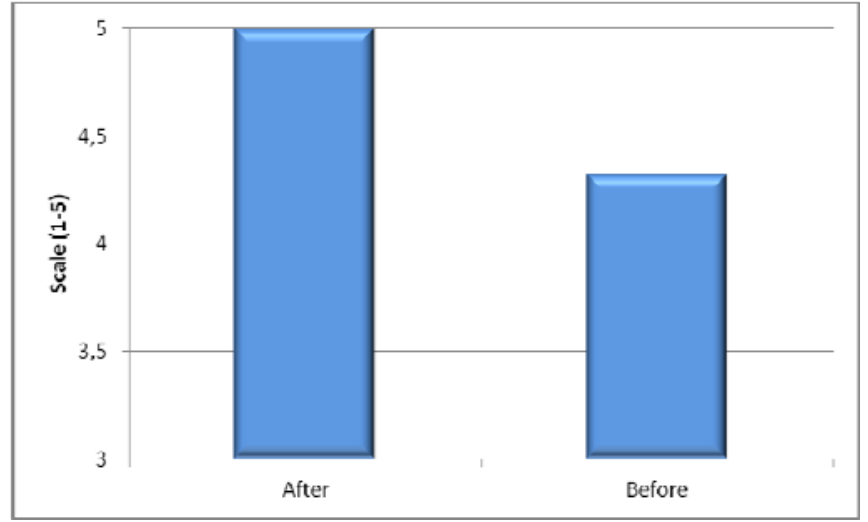

Fig. 9. Evaluation of the item "Lesson preparation" on a 1-5 scale.

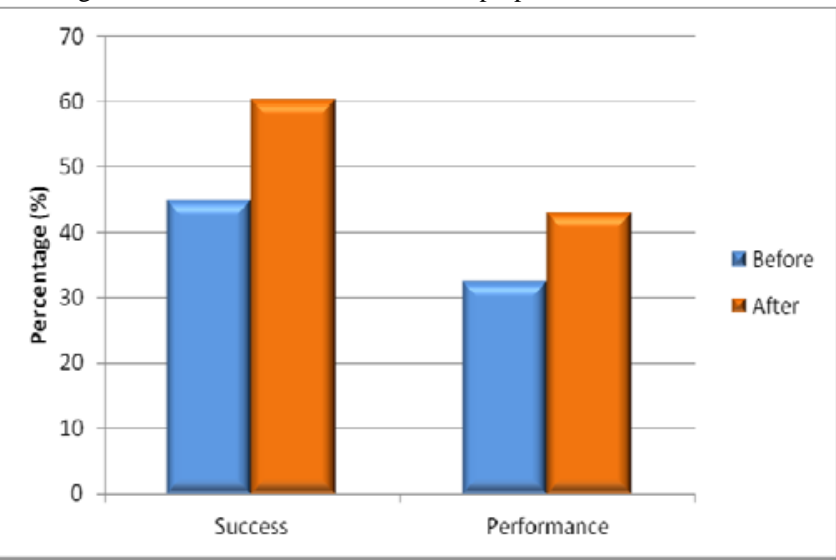

Fig. 10. Percentage of enrolled students who passed the course (performance rate) and percentage of students' following the course who passed it (success rate).

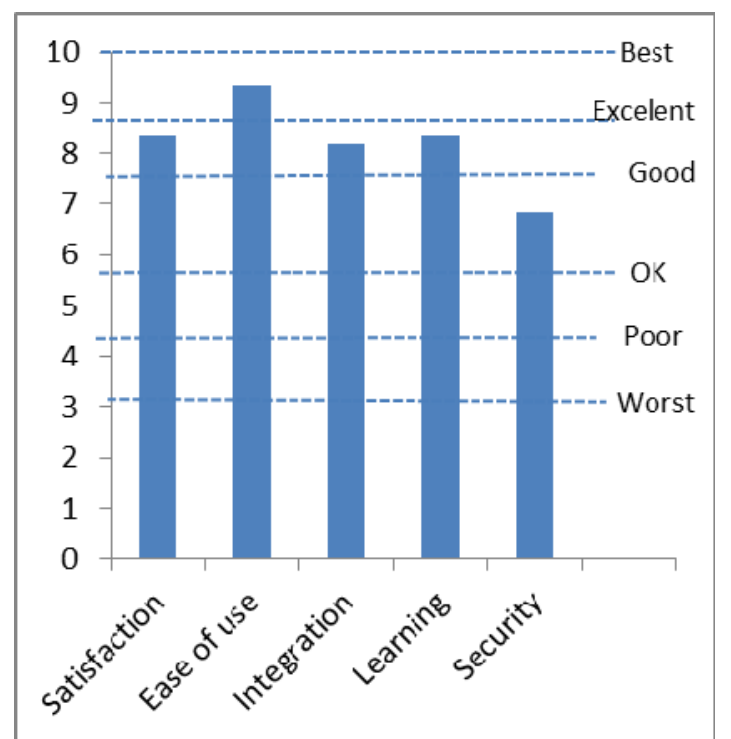

Fig. 11. Results of the usability analysis conducted by the pilot teachers.

\section{CONCLUSIONS AND FUTURE WORK}

The application of the code of good teaching practice in daily activity is a complex task since it involves many processes and requires the documentation of activities. In order to ease its use, a Web software tool that supports the 
application of the code has been presented. This tool is intended to systematize and facilitate the real-world application of the code at the university level.

This paper shows the results of using this code in a particular course. For this purpose, several aspects regarding students' satisfaction and academic results have been evaluated before and after applying the code. For this evaluation, objective external metrics have been used. Results of the evaluation show that all examined items improved after applying the code. As the code covers all aspects of the teaching process and follows quality and innovation standards, it is possible to state that it helps to systematize educational activities by applying good practices. The efforts performed had a positive effect on the teaching practice, which was perceived by students. Proof of this is that both satisfaction metrics and academic results clearly improved.

From the teachers' point of view, an important feature of the code is that it allows high levels of customization both in selecting the processes to be implemented and in selecting the time to fill in the records. In this way, the active participation of teachers is encouraged during use. Thus, the tool implemented should evolve in the future towards the interconnection between teachers, which could have a positive influence on the degree of teachers' participation. In this regard, the platform should enable the exchange of custom templates and records between teachers as well as use their experience. In order to improve the teachers' perceived security of the platform, explanatory messages could be included in order to guide teachers during use.

A preliminary application of the code has been conducted in the course "Fundamentals of Digital Systems". This is a starting point. As a future line of work, we expect to apply it to other courses in several academic areas, and to increase the number of teachers involved in the use of the Web application in order to have a larger test group.

Another effort to be made in the near future is the generation of the records to be filled in by teachers in the Reusable Learning Objects (RLOs) format and support such records. A RLO can be defined as a digital resource designed in order to be reused by any interested teacher around the world. In this way, both optimization of efforts and knowledge sharing are promoted. It is also remarkable that the Web application supports documents in popular formats (doc, docx, pdf, odt, ods, xls, etc.) which do not have to follow a standardized structure. The future final goal is that teachers can share their own records with the entire educational community, promoting the spread and consolidation of the code as an integral educational tool based on quality and innovation.

\section{ACKNOWLEDGMENTS}

We thank the "European Social Fund" and the "Departamento de Innovación, Investigación y Universidad" (Gobierno de Aragón) for their support.

\section{REFERENCES}

[1] M. Palani Natha Raja, A. M. Abirami, "Performance Excellence Model for Engineering Education: An Enabler for Sustaining Quality,” Journal of Enginnering Education Transformations, vol. 28, 2015, p. 122-130

[2] K. Ishikawa, ¿Qué es el control total de calidad?: La modalidad japonesa. Editorial Norma S.A., 1997.

[3] J. Anttila, "Quality and Innovation," in 57th European Organization for Quality Congress. Quality renaissance. Co-creating a viable future, Tallinn, Estonia, 2013, June 17th-20th.

[4] I. Plaza, F. Ibáñez, R. Igual and F. Arcega, "Use of quality standards as element of innovation in Higher Education," in The 4th Iberian Conference on Information Systems and Technologies, Povoa de Varzim, Portugal, 2009, pp. 567-572.

[5] R. Igual, I. Plaza, F. Ibañez, C. Medrano and F. Arcega. "Quality and innovation. Web-based code of good teaching practice.” In The 3rd Workshop on Interaction Design in Educational Environments, Albacete, Spain, 2014, June 9th.

[6] T. Rexwinkel, J. Haenen and A. Pilot, "Quality assurance in higher education: analysis of grades for reviewing course levels.” Quality \& Quantity, vol. 47, pp. 581-598, doi: 10.1007/s11135-011-9481-6.

[7] J. B. Biggs, Teaching for quality learning at university: What the student does. UK: McGraw-Hill Education, 2011.

[8] S.H. Chen, "The establishment of a quality management system for the higher education industry.” Quality \& Quantity, vol. 46, 2012, pp. 12791296, doi: 10.1007/s11135-011-9441-1.

[9] [9] N. Jethro, “A Tale of Two Qualitys: Reflections on the Quality Revolution in Higher Education," Quality in Higher Education, vol. 16 no. 1, 2010, pp. 51-53.

[10] H. Pratasavitskaya and B. Stensaker, "Quality Management in Higher Education: Towards a Better Understanding of an Emerging Field,” Quality in Higher Education, vol. 16, no. 1, 2010, pp. 37-50.

[11] D. Laurillard, Rethinking university teaching: A conversational framework for the effective use of learning technologies. England: Routledge, 2013.

[12] Y. LiFeng, "Reform of Basic Computer Education and Cultivation of Innovation Talent in Higher Education.” Advances in Computer Science, Environment, Ecoinformatics, and Education Communications in Computer and Information Science, 2011, pp. 217: 499-503, doi: 10.1007/978-3-642-23339-5 91

[13] Y. Changli and J. Hongchun, "On the Cultivation of Innovative Talents in Colleges and Universities,” International Education Studies, vol. 2, no. 4, 2009, pp. 162-167.

[14] H-Y. Wu, J-K. Chen and I-S Chen, "Ways to promote valuable innovation: intellectual capital assessment for higher education system.” Quality \& Quantity, vol. 46, no. 5, 2012, pp. 1377-1391.

[15] I. Plaza, R. Igual, C. Medrano and M.A. Rubio, "From Companies to Universities: Application of R\&D\&I Concepts in Higher Education Teaching.” IEEE Transactions on Education, vol. 56, no. 3, 2013, pp. 308-315.

[16] Gh. Militaru and C.I. Dumitrescu, "Higher education: aspects of quality and innovation in an era of global changes.” In The 6th International Conference on the Management of Technological Changes, Alexandroupolis, Grecia, 2009, September 2nd-3rd.

[17] E. Van Kemenade and P.L. Rinderu, "A comparison algorithm of quality assurance \& accreditation in eastern European countries higher education systems - aspects related to the organizational culture and innovation of HEIs." in The 6th International Conference on the Management of Technological Changes, Alexandroupolis, Grecia, 2009, September 2nd-3rd.

[18] P. Hines and D. Taylor, Going lean. Cardiff, United Kingdom: Lean Enterprise Research Centre, 2000.

[19] J. Holmes, Struts: The Complete Reference. New York: McGraw-Hill Osborne Media, 2006.

[20] EUPT, "Escuela Universitaria Politécnica de Teruel (España)," Accesible in http://eupt.unizar.es/, 2016, February, 28th.

[21] A. Bangor, P.T. Kortum y J.T. Miller, “An empirical evaluation of the System Usability Scale.” International Journal of Human-Computer Interaction, vol. 24, no. 6, pp. 574-594.

Esther Sainz-Martin received the B.Sc. in Electrical Industrial Engineer (1996) and the M.S degree in Industrial Engineering (2003) from the 
University of Zaragoza. She currently works as Lecturer in the Department of Electrical Engineering at the School of Engineering and Architecture (EINA) of the University of Zaragoza. She is a researcher of the EduQTech group. Her main research interest is the application of an innovative culture based on quality concepts in all areas of university education.

Raul Igual-Catalán received the M.S. degree in Electronics Engineering (2010) and the Ph.D. in Electronics Engineering from the University of Zaragoza, 2014. He worked as associate professor at the University of Zaragoza (2013-2015) and he is currently a Lecturer in the Department of Electrical Engineering. His main research interests include the use of sensors for quality of life and health applications, the bioelectricity and new technologies in higher education.

Inmaculada Plaza-García (M’02-SM’06) received the M.S. degree in physics, Advanced Studies Diploma (DEA) in design and manufacturing engineering, and Ph.D. degree in electronic engineering from the University of Zaragoza, Zaragoza, Spain, in 1994, 2001, and 2005, respectively. She is currently a Senior Lecturer (TU) with the Electronics Department, Polytechnic University School of Teruel, University of Zaragoza, Teruel, Spain. Her research interests include quality in R\&D\&I activities, quality of life, and quality in education. Along with Dr. Francisco Arcega, she is the Coordinator of the research group EduQTech. Dr. Plaza is currently Past-Chair of the Spanish Chapter of the IEEE Education Society.

Iván García-Magariño obtained the Ph.D. in Computer Science Engineering in 2009 from the Universidad Complutense de Madrid. From 2010 to 2014, he worked as Assistant Professor at the Open University of Madrid. Currently, he works as lecturer at the University of Zaragoza. He has published articles in journals with high impact such as "Information Sciences" and "Engineering Applications of Artificial Intelligence”. Its main area of interest is agent-based simulators and new technologies in education.

Francisco Ibáñez-Álvarez received the B.Sc. in Electrical Engineer from the University of Zaragoza (1982) and the M.S. degree in Industrial Organization Engineering from the University of Vic (2005). He has been working in teaching environments for more than 30 years in several companies and institutions. He currently works as Professor in the Department of Electrical Engineering at the University of Zaragoza. His main research interests are quality of R\& D+I activities focusing on the process and the product as well as quality in education.

Francisco Javier Arcega-Solsona received the M.S degree in Physics from the University of Zaragoza in 1976 and the Ph.D. degree from the same university in 1981. He held the position of assistant professor in the Department of Electrical and Electronics from 1976 to 1983. He is currently Professor in the area of Electrical Engineering at the University of Zaragoza. F.J. Arcega is member of IEEE, reaching the rank of Senior Member in 2005. He regularly collaborates with the Power Electronics Society and the Education Society. 